\title{
A NEW CONCEPTION OF THE ELEMENTS OF SENSATION.
}

BY JOHN S. B. STOPFORD, M.D.

Professor of Anatomy, University of Manchester.

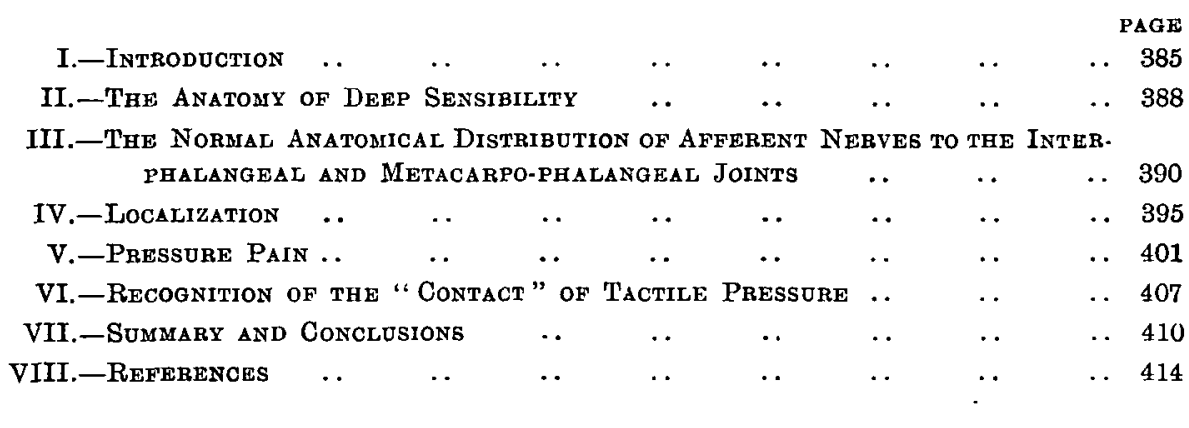

\section{I.-Introduction.}

We owe to Head our deliverance from the chaos which existed with regard to the afferent nervous system up to the time of the publication of his reconsideration of the distribution and function of the peripheral nerves. By means of investigations by measurable tests of each form of sensation, he was able clearly to demonstrate that what had been termed "diminished sensibility" was really a state in which some forms of sensation had been lost whilst others were retained. He was able to prove conclusively that "parts which have universally been considered to be areas of diminished sensibility turn out to be totally insensitive to certain higher forms of stimulation." In the study of the recovery of cutaneous sensation after suture of a peripheral nerve, he discovered two stages. During the earlier stage there was a gradual diminution in the area insensitive to pain produced by the prick of a pin, and to heat and cold, but the recovered sensibility was appreciably altered. The prick evoked a sensation which radiated widely and caused unnatural discomfort, and although the extremes of temperature could be appreciated no sensation of heat or cold was induced by intermediate thermal degrees. During the later or second stage of recovery the affected part began to become sensitive to light touch and 
to degrees of temperature which induced sensations of "warm" and "cool." With further improvement it became possible to discriminate two points applied simultaneously, and the characteristic radiation of the first stage gradually disappeared as more accurate cutaneous localization returned.

Such observations during recovery after suture of a peripheral nerve demonstrated the existence of two systems in cutaneous sensibility, which Head named the protopathic and epicritic, and it was thought that the former system regenerated more rapidly and with greater ease than the latter. The crucial test of this division into two systems was the classical experiment upon Head's own arm. In this experiment the radial nerve was divided at its origin from the musculospiral, and the musculo-cutaneous nerve at the point where it perforated the deep fascia, just proximal to its division into the two branches which supply respectively the anterior and posterior aspects of the preaxial half of the forearm. Some days later it was discovered that, in addition to the larger area in which, except for the dissociation at the edges, there was a complete loss of cutaneous sensation, there existed a small triangular portion where normal sensation to light touches with cotton wool and Von Frey's hairs was retained, but there was insensitiveness to the prick of a pin. The dissociation of the sensation of this triangle was the reverse of that found at the periphery of the larger area, where the prick of a pin was appreciated but there was no appreciation of light touches with cotton wool. The prolonged investigations of these complementary areas of dissociated sensibility established the existence of the two systems in cutaneous sensibility, and proved that these two systems were independent and did not depend upon simply a modification of the same system of nerve fibres and end organs.

To these two systems found in cutaneous sensation Head added a third, deep sensibility, which was capable of responding to pressure, passive movement of joints, and pain caused by excessive pressure. He found that a part endowed with only this third form of sensibility possessed not only the ability to recognize a pressure stimulus, but also the power to localize it with remarkable accuracy. Furthermore it was decided that "the fibres subserving this form of sensation run mainly with the motor nerves, and are not destroyed by division of all the sensory nerves to the skin." Our knowledge of deep sensibility is based almost entirely upon an investigation of the residual sensation in the experiment upon Head's own arm. The nerves divided in this 
experiment were selected because it was thought that, since these nerves were usually considered as purely cutaneous in distribution, a large area of skin would be rendered completely insensitive and yet the sensibility of the subcutaneous tissues would be undisturbed. Observations will be advanced in this paper to show that it is anatomically impossible to isolate deep from cutaneous sensibility, and that, by employing methods which Head has used for the investigations of cutaneous sensation, it is possible to find after division of peripheral nerves at various levels most intricate types of dissociation in the various forms of sensibility grouped as "deep." Furthermore, having obtained an insight into the production of these dissociations, it is practicable during regeneration after suture to recognize two systems in deep sensibility.

My attention was directed to this subject during the progress of a study of the late results of secondary suture of certain peripheral nerves of the upper limb [7] -as the median and ulnar-since it became apparent that the sensory condition found did not conform with our present conception of the subject. The chief discrepancy had reference to deep sensibility, and most of the investigations recorded in this paper are concerned with this aspect of sensation. It was discovered in some patients, after suture of the median, that all the muscles-when tested individually - had recovered voluntary power and yet the hand was of slight practical service when the patient attempted purposive movements necessary on his return to work. These patients complained that they lost the grip of their tools, and on further inquiry it was found that they could use the hand fairly well as long as they concentrated upon the movements, but it failed to function satisfactorily as soon as they ceased to watch what it was doing. Several volunteered the information that the hand was useless in the dark or when they were unable to observe what they were trying to do with it. Such complaints were made by patients some three or more years after the performance of end to end suture, even when the cutaneous sensation had made a fair recovery and all the muscles, when tested individually, exhibited good voluntary power. It seemed clear that such a disability could only be due to a loss of afferent stimuli from joints, muscles, tendons and other deep structures. It was only practicable to investigate directly the extent of the recovery of fibres conveying sensory impressions from such deep structures as the joints. An investigation as to the sense of posture and the appreciation of passive movements in the finger and thumb articulations supplied by the nerve was carried 
out and it was discovered that these were generally lost, or at the best very defective, even when fully three years had elapsed since the time of the suture. This loss of appreciation, or defective recognition, of passive movement in the joints supplied by the nerves was found irrespective of the level of the suture; although, according to Head, there would be no serious loss of deep sensibility after section of the median or ulnar nerves low down, e.g., at the wrist. This inquiry into the recovery after end to end suture of afferent fibres from joints gradually directed me to the investigations recorded in this paper.

\section{II.-The Anatomy of Deep Sensibility.}

By the subdivision of sensation into cutaneous (epicritic and protopathic) and deep, we are at once confronted with certain anatomical difficulties, which are emphasized in the hand and digits where much of the experimental work upon sensation has been performed. We cannot separate anatomically the fibres distributed to cutaneous and subcutaneous structures of any sensory nerve of sufficient size to warrant its use as a source of information about sensation. No nerve trunk or branch, of sufficient size to receive a name, has an exclusively cutaneous distribution. My own clinical investigations prove that the radial [8] besides supplying skin is distributed to certain articular structures, and careful dissection of nerves which have unfortunately been described as "purely cutaneous" will show that a large proportion of fibres terminate in the subcutaneous tissues. Of course such a gross method of investigation as dissection does not give us any information about the function of these fibres which can be traced into deeper structures, but in view of the clinical findings after division of the radial nerve (which is usually designated a purely cutaneous nerve), we must not assume that any nerve, of sufficient size to justify its selection for experimental work upon sensation, is purely cutaneous until it has been proved. In the course of this work the extreme difficulty of determining clinically whether the sensory fibres of a nerve are distributed solely to skin or not has become manifest, the chief hindrance arising from the fact that we cannot ascertain for certain whether some aspects of sensation are subserved by fibres terminating in skin, or subcutaneous tissues, or both. This difficulty is well illustrated by localization. From the experiments upon localization it is clear that fibres subserving this form of sensibility terminate cutaneously and subcutaneously. It seems possible from my own observations and those of others that discrimination may he also served by both cutaneous and subcutaneous systems, 
but there are even greater technical difficulties to be overcome in making a decision with regard to this form of sensation. At first sight the experiment upon Head's own arm seems to establish discrimination as purely dependent upon a cutaneous supply, but it depends upon the assumption that the radial and the terminal branches of the musculocutaneous are purely cutaneous nerves, and since in a larger proportion I have found this not to be the case, the results cannot be accepted as proof that discrimination is only a cutaneous form of sensation. In the case of division of the median and ulnar it is clearly quite impossible to advance any proof with regard to this question owing to the complex anatomical distribution to subcutaneous tissues in this region. Whereas we know accurately the normal cutaneous distribution of the median and certain variations [6] in its cutaneous supply, we find that the corresponding deeper tissues receive sensory fibres from at least three sources. The subcutaneous structures of the palmar surface of the thumb, index and middle fingers are supplied from the median nerve direct by means of the digital branches, and from branches arising from the main trunk proximal to the wrist which probably run chiefly with the muscular branches, and so pass to their distribution along tendons. The subcutaneous tissues of the palm corresponding to the cutaneous distribution of the median are supplied, first by branches from the nerve itself as it breaks up in the palm, secondly by branches which pass down with the long tendons, and thirdly from the deep branch of the ulnar or perforating branches of the radial or both. The latter two possible sources of sensory fibres are shown by the fact that even after division of the median nerve in the upper arm, pressure and often pressure-pain can be recognized over the whole of the palm in some patients, and in all over a considerable part of the palm; and similarly after division of both the median and the ulnar in the arm contact and pressure pain are appreciated over part of the palm, especially on the outer side.

The distribution to subcutaneous tissues in the case of the ulnar is not quite so complex, but sufficiently intricate to make it impracticable to isolate it from the cutaneous supply. In the hand deep sensibility is only in part subserved by fibres which run with muscular branches, since the digital nerves-which are generally regarded as cutaneousdefinitely supply the articulations [8]. One form of sensation included under the term of deep sensibility is therefore invariably affected by division of the median or ulnar, whether the section of the nerve occurs at the wrist or in a more proximal part of the limb. 
The complex anatomical arrangement of the sensory supply to the subcutaneous tissues of the hand makes it exceedingly difficult to investigate what has been termed deep sensibility; it is not accurate to regard it as the residual sensation after division of a purely cutaneous nerve unless we are certain that a nerve so designated has solely a sensory distribution to skin, and from the results recorded in this paper I maintain that we cannot so adjudge the radial nerve. Most anatomical text-books describe the radial as "purely" or "solely" cutaneous, but it seems evident that in the past anatomists have designated nerves as purely cutaneous on insufficient evidence. This appears to have misled Head and his co-workers into the belief that in "a human experiment in nerve division" only cutaneous sensation was destroyed, and deep sensibility persisted undisturbed.

From these conclusions it would appear that any division of sensation into cutaneous and deep must be regarded as artificial, and is apt to be misleading when applied to clinical work owing to the complex anatomical distribution of sensory fibres to the subcutaneous tissues.

ili.-The Normal Anatomical Distribution of Afferent Nerves to the Interphalangeal and Metacarpo-phalangeal Joints.

After a few patients had been examined with reference to the extent of the recovery of sensory fibres supplying the articulations of the digits, a difficulty presented itself owing to our imperfect knowledge of the normal distribution of peripheral nerves to the interphalangeal and metacarpo-phalangeal joints. . Consequently it became necessary to discover precisely the normal distribution of the various nerve trunks to the joints, in order to be able to estimate accurately the recovery which had occurred. The details of this part of the work have already been fully recorded in a recent paper [8] and it is necessary only to summarize the conclusions bearing upon deep sensibility.

In all fifty-one patients were examined and they were selected because it was known that a complete division of definite peripheral nerves of the upper extremity existed. Any patient manifesting such complications as division of tendons or limitation of mobility of joints was disregarded for obvious reasons.

The examination was first made on the normal side in order to make the patient quite familiar with the method and what was expected of him; secondly to provide a standard and control for comparison; 
and thirdly to enable me to test the reliability and attention of the patient. It was surprising how few patients had to be discarded as. unreliable when sufficient time was taken to make quite clear to them what was wanted, and enough patience was expended. A relatively large number, who were otherwise suitable, had to be omitted on account of arthritic adhesions. Each segment of the digit, above and below the joint to be examined, was grasped firmly by the lateral borders and then the joint was gradually and evenly flexed or extended. The segments of the digit must be grasped firmly, as Head [3] has pointed out, so that the additional pressure required to move the joint shall not be distinguishable and thus lead to the recognition of movement from impressions other than those arising from the articular structures. The movement of the joint must also be made as far as possible at a uniform rate and not too slowly. The patient was requested to state when he first felt any movement, and on answering was further asked at which joint.it occurred, and also the apparent direction of the movement. The latter two questions were soon found to be of fundamental importance for any accurate investigation of the appreciation of passive movement in a joint, since it was discovered that patients were able frequently to recognize movement of a particular digit without possessing any knowledge of the joint at which it occurred or the direction in which it moved. Quite early in the investigation it became manifest that this recognition of mere movement of a finger was. obviously dependent upon alteration in tension or position of the tendons (and consequent stimulation of the nerve endings in relation to them), and did not arise from any stimulation of afferent terminals in the bones, cartilage, and capsule of the joint. Several patients informed me that they felt the movement of something at the wrist and knew it was pulling on a finger, but were quite unable to discover from the sensation aroused at which joint the movement took place. Fortunately this source of fallacy was discovered at an early stage in the investigation, and errors arising from it were consequently prevented. From a review of other work on this aspect of sensation it seems possible that fallacies may have arisen in the past from this cause.

Another source of possible error, which was soon determined, arose from the difficulty patients had in expressing the joint at which the movement was experienced. Such a term as "first joint" was apt to be misleading, since some referred this to the metacarpo-phalangeal articulation, and others to the distal interphalangeal. Consequently it was found necessary at the outset of each examination to have a clear 


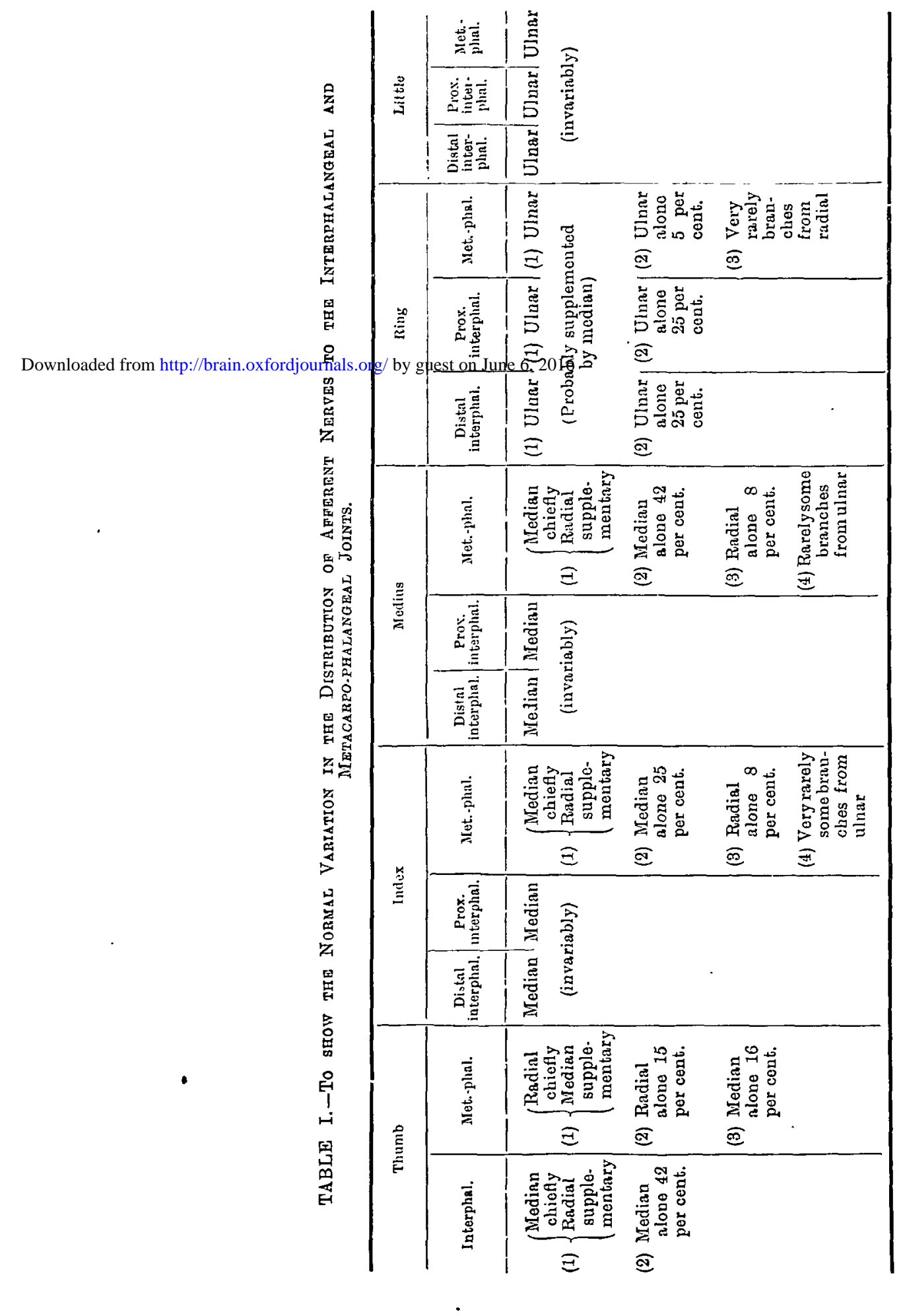


understanding with the patient as to the terminology he adopted for the various joints. The results of this anatomical inquiry into the normal supply of the afferent fibres to the interphalangeal and metacarpophalangeal joints are summarized in Table I, but it must be understood that the percentages there stated can be regarded as only approximate, since only fifty-one patients were examined.

The most surprising result found in these examinations was that no difference in the extent of the loss of the recognition of posture and appreciation of passive movement was produced by section of the nerve under inquiry at different levels in the limb, a precisely similar distribution being found whether the nerve was severed in the upper arm, forearm, or at the wrist. In seven patients (median four, ulnar two, median and ulnar one), the division was at the wrist, and there had been no division of tendons, yet the loss of appreciation of passive movement was just as widespread and as profound as when the nerves had been divided in the upper part of the forearm. In twenty others the division had occurred in the forearm, and in a number of these in its distal part. The patients in the latter category did not manifest any loss of the recognition of pressure, yet the loss of appreciation of posture was just as extensive as after section in a more proximal part of the limb, even where one or more digits were completely anæsthetic to all forms of stimulation.

It is of particular importance to notice the condition found in fourteen patients in which the musculo-spiral had been severed, since the study of these patients really resolved itself into an investigation of the distribution of the radial and lower external cutaneous branches of this nerve to the joints. In two the recognition of passive movements appeared to be perfect in all the joints, but in the other twelve one or more articulations of the thumb or fingers were affected, and the extent of this distribution was found to be subject to considerable variation. It will be remembered that there was no disturbance of the perception of passive movement in Head's historical experiment upon which depends our present knowledge of deep sensibility; and it seems possible, from the fact that a similar result was found in only two patients out of fourteen, that this form of distribution is the exception rather than the rule. Furthermore in view of the evidence of the very frequent presence of sensory fibres in the radial for the supply of certain articulations of the digits, it is no longer possible to regard the radial as a purely cutaneous nerve.

In the course of this study a very intimate relationship between 
the variations in the cutaneous (epicritic) and articular distributions of the various nerves was observed, and this is illustrated in fig. 1 :-

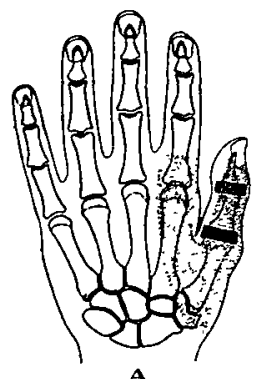

A

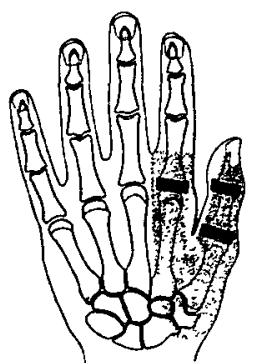

B

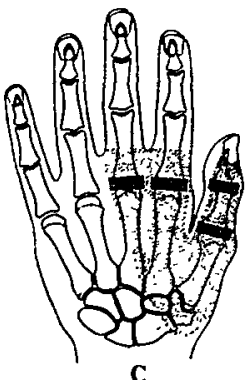

C

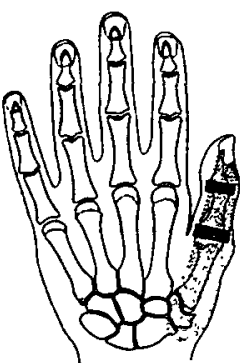

D

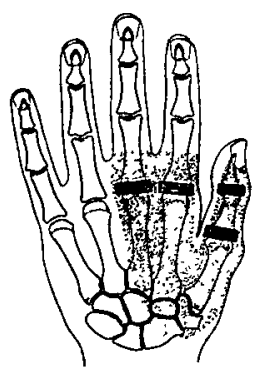

$\mathbf{E}$

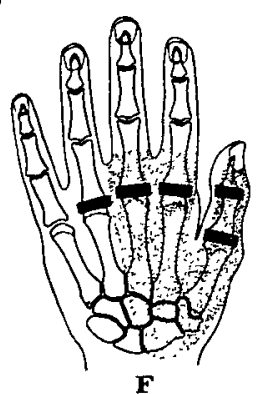

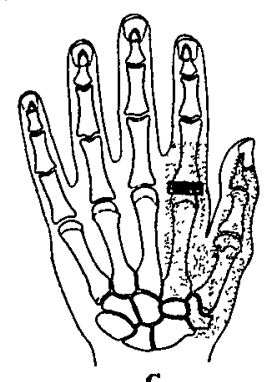

G

- Complete loss of appreciation of passive movement. $=$ Impaired recognition of passive movement.

Extent of anæsthesia to lightest possible stimulations.

$$
\begin{aligned}
& \text { Type } A=1 \text { patient. } \\
& \text {, } B=4 \text { patients. } \\
& \text { " } C=3 \text { patients. } \\
& \text { " } D=1 \text { patient. } \\
& \text { Type } E=1 \text { patient, } \\
& \text {, } F=1 \text { patient. } \\
& \text { " } G=1 \text { patient. }
\end{aligned}
$$

FIG. 1.-To show the intimate relationship between the cutaneous (epicritic) and articular distributions of the radial nerve.

The loss of the appreciation of passive movement in the joints of the digits which exhibit no anæsthesia to tactile pressure is at first sight rather mystifying in view of our present conception of the pathway for the deep afferent impressions, i.e., along nerve-fibres which run chiefly with muscular branches and so reach the articulations by the way of such channels as tendons and blood-vessels. This view must largely disregard the digital branches of such nerves as the median and ulnar as a source of articular branches to the interphalangeal and metacarpo-phalangeal joints, and yet a simple dissection of these branches will demonstrate clearly fine filaments which pass to the periosteum of the phalanges and to the interphalangeal articulations. It may be presumed that these branches from the digital nerves are either afferent or vasomotor in function. The results found in the 
clinical examinations just recorded of patients suffering from division of the median or ulnar at the wrist, uncomplicated by any injury to the tendons, appear to show conclusively that some of these filaments are afferent in function and transmit information which enables us to appreciate the site, direction, and range of passive movement. Since the extent of the loss of appreciation of passive movement was not altered by the anatomical level of the section of the nerve, it appears that few, if any, fibres subserving this form of deep sencibility run with the branches to forearm muscles, the tendons of which are inserted into the phalanges. Several patients suffering from division of the median or ulnar at the wrist were able to recognize "movement" of a particular finger, probably as a result of alteration of tension or position of the long tendons; but it was clear that this knowledge was not produced by stimulation of afferent terminals in the neighbourhood of the joint, since some of them explained that they felt "the pull at the wrist," and so "guessed a finger was being moved." None of these were able to recognize at which joint the movement occurred, or in what direction it was made.

These conclusions have been verified upon a number of patients examined three or more years after suture of the median or ulnar nerve at the wrist, who at the best still exhibited very defective recovery of the appreciation of passive movement in the joints supplied by the affected nerve. From these investigations it appears that the digital nerves are the only important source to the joints of branches which transmit impulses that enable us to recognize position and movement.

On applying this knowledge to the examination of large numbers of patients upon whom end to end suture of the median, ulnar, or musculospiral nerves had been performed, it was found that this form of sensation, irrespective of the level of the suture, failed to show any signs of recovery until a very late stage-a time when there was evidence of return of epicritic sensibility.

\section{IV.-TJOCALIZATION.}

The findings with regard to spacial recognition in three dimensions led me to investigate other aspects of deep sensibility.

At present it is believed that pressure is localized with remarkable accuracy even when there is a complete lose of cutaneous sensibility, and it is surmised that this form of sensation is dependent upon 
afferent fibres which run in muscular, articular, and subcutaneous: branches.

Hitherto, no perfectly satisfactory method for testing accurately tactile localization has been evolved. Most methods now in use only demonstrate very gross defects, and fail to furnish measurable results. The tests most frequently adopted in the investigation of tactile localization only show such a gross error as failure to recognize correctly the segment of the digit upon which the pressure has been applied-a defect which I have found to occur several times in apparently normal individuals [9]. Consequently an effort was made to discover some method which would supply more accurate information about the power of localization, and also provide measurable results. The method found to be most serviceable and practicable was a slight modification of that described as Henri's, but, unfortunately, it has not. been possible for me to procure the original description of his method. The patient, having the part to be tested obscured from his vision, marked upon a life-sized diagram the position of the point judged to be stimulated, whilst the observer recorded on another similar diagram the exact point touched. After a sufficient number of points had been localized, the two diagrams were superimposed and the two points recorded in each test were marked on one diagram-in different colours or by distinctive signs, and then the error in each case was measured off with a millimetre scale. From these measurements, if a sufficient. number of points had been localized, the average error for the whole or individual parts-as each digit-could be determined. In the latter part of the investigation only one chart was used, the observer recording the exact point stimulated after the person examined had marked the point where he felt the stimulation; and this simpler procedure proved as successful, and apparently as reliable, as the more tedious original form.

Using this method, which possesses the great advantage of giving measurable results, the following experiments were performed:-

Experiment 1.-This series, in which ten members of the medical profession provided the subjects for examination, was undertaken at the outset for two principal reasons. In the first place it was thought that any possible fallacy due to unfamiliarity with the diagrams would be reduced to a minimum; and, secondly, such a series would provide valuable information with regard to the introspective aspect of this form of sensation. The tactile pressure was made by a blunt instrument about $0.2 \mathrm{~cm}$. in diameter. The palmar and dorsal surfaces of 
each hand and digit were explored in turn. Twelve stimulations were made on each surface, eight or nine of which were on the digits and the rest on the palm or dorsum of the hand. The average error for the ten subjects was found to be $0.6 \mathrm{~cm}$. for the palmar and $0.5 \mathrm{~cm}$. for the dorsal surfaces of the right hand and fingers, and $0.5 \mathrm{~cm}$. for both surfaces of the left hand and fingers.

Experiment 2.-In this series, ten non-medical people were examined and only the palmar surface of the two hands and digits explored. The ten were composed of clerks, departmental stewards, porters, and second-year students. The same number of stimulations were made, and the same proportion on the hand and digits, by the same instrument as in the previous experiment. The average error for the ten was $0.6 \mathrm{~cm}$. for the right hand and $0.7 \mathrm{~cm}$. for the left. The close similarity in the results in the two experiments is rather remarkable, and shows that any error due to unfamiliarity with the charts, at any rate for the palmar surface of the hand and digits, must be very slight. This conclusion was confirmed by a large series of tests carried out upon pensioners under my charge, in whom an average error of $0.6 \mathrm{~cm}$. was found for the palmar surface of the hand and digits on both sides.

Experiment 3.-This series was undertaken to discover the normal average error for the individual fingers and to find out if there was normally any definite variation in the power of localization in the different fingers. Two people were chosen from each of the former experiments and twelve pressure stimulations (six on the palmar and six on the dorsal surface) were made on each finger of right hand. As far as possible two stimulations were made on both surfaces of each segment of a finger.

The average error for the four persons examined was found to be $0.4 \mathrm{~cm}$. for the index, middle, and ring fingers, and $0.3 \mathrm{~cm}$. for the little finger; from which it appeared that the power of localization did not vary to any obvious extent in the four fingers.

Further details and tables showing individual.errors in these three experiments are given in the paper previously referred to.

Experiment 4.-From the results which were being found on applying this test to patients suffering from division of peripheral nerves, it was decided to perform a series oî tests using as a stimulus a hair, which could not possibly stimulate any but cutaneous nerve terminals. For this investigation three medical people were chosen from those used in Experiment 1 and a similar number from the non-medical group of 
Experiment 2. The same number of stimulations were made with the hair as in the former experiments where pressure had been used as the stimulus, and the conditions under which the tests were performed were identical with those of the previous experiments. It was found that a purely cutaneous stimulus could be localized very accurately; in Table II the results are compared when using a hair and when using pressure with an instrument $02 \mathrm{~cm}$. in diameter. The latter records had been taken some weeks previously.

TABLE II.

\begin{tabular}{|c|c|c|c|c|c|c|c|c|c|}
\hline \multirow[b]{3}{*}{ SL. } & \multicolumn{4}{|c|}{ Right Hand } & & \multicolumn{4}{|c|}{ Left Hand } \\
\hline & & air & Pre & sure & & $\mathrm{H}$ & & Pres & ssure \\
\hline & $\cdot 5$ & $\mathrm{~cm}$. & $\cdot 4$ & $\mathrm{~cm}$. & .. & $\cdot 7$ & $\mathrm{~cm}$. & & $\mathrm{cm}$. \\
\hline SJ. & $\cdot 5$ & , & $\cdot 7$ & ", & .. & $\cdot 7$ & ", & -5 & ," \\
\hline EAL. & $\cdot 6$ & , & $1 \cdot 0$ & , & .. & .5 & , & -8 & 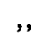 \\
\hline AECD. & $\cdot 5$ & , & .5 & , & .. & $\cdot 4$ & , & $\cdot 6$ & ," \\
\hline$A G$. & $\cdot 6$ & ", & -7 & , & .. & $\cdot 7$ & ", & $\cdot 7$ & , \\
\hline RE. & $\cdot 5$ & , & $\cdot 5$ & , & .. & $\cdot 5$ & ", & $\cdot 5$ & , \\
\hline Average & $\cdot 5$ & ", & $\cdot 6$ & , & . & $\cdot 6$ & , & $\cdot 6$ & , \\
\hline
\end{tabular}

From these results it will be seen that accuracy of localization is practically the same, for either a purely cutaneous stimulus or pressure, in individuals with normal sensation.

It is of interest to notice that in the observations made upon Head's hand [5], it was found that accurate localization of cutaneous stimuli did not return until the skin was sensitive to tactile hairs after shaving.

Experiment 5.-After the former more or less preliminary experiments, which were undertaken chiefly to gain experience and establish the normal degree of error in localization, had been carried out, the pressure test was applied to twenty patients who were known from surgical onservations to have suffered from division of one or two peripheral nerves of the upper limb at various levels. A similar number of stimulations as in Experiments 1 and 2 were made over the region of the cutaneous distribution of the affected nerve and, for comparison, on the normal part of the same hand or a similar region on the unaffected hand. Stimulations were made with the same instrument as that used in the other experiments. In ten patients, the observations were taken shortly after the performance of secondary suture of the injured nerve, at a time too early for any regeneration to have occurred. The results are tabulated in Table III.

The average error for the ten patients over the cutaneous field of the sutured nerve was $1.4 \mathrm{~cm}$., whereas on the normal part it was $0.6 \mathrm{~cm}$. 
Table III.-Localization (Pressure) Tests, amter Complete Nerve Division and Suture at a Tiame when no Regeneration ilad occurred.

\begin{tabular}{lllcc} 
Patient & \multicolumn{1}{c}{ Nerve } & \multicolumn{1}{c}{ Site of suture } & $\begin{array}{c}\text { Average error for } \\
\text { localization over area } \\
\text { of epieritic loss }\end{array}$ & $\begin{array}{c}\text { Average error for } \\
\text { localization over } \\
\text { normal area }\end{array}$ \\
T. E. & Median & Upper forearm & $1 \cdot 2$ & $\cdot 6$ \\
J. D. & Median & Middle of forearm & $1 \cdot 4$ & $\cdot 5$ \\
H. S. & Musculo.spiral & Elbow & $1 \cdot 1$ & $\cdot 4$ \\
T. M. & Ulnar & Middle of forearm & $1 \cdot 7$ & $\cdot 7$ \\
A. M. & Median & Middle of forearm & $1 \cdot 0$ & $\cdot 5$ \\
N. D. & Ulnar & Middle of forearm & $1 \cdot 2$ & $\cdot 4$ \\
J. J. T. & Median & Arm & $1 \cdot 8$ & $\cdot 6$ \\
A. L. B. & Ulnar & Elbow & $1 \cdot 1$ & $\cdot 6$ \\
J. T. S. & Ulnar & Lower forearm & $1 \cdot 9]$ & $\cdot 8$ \\
F. S. & Ulnar & Middle of forearm & $1 \cdot 2$ & $\cdot 8$
\end{tabular}

In the case of T.M. two stimulations on the little finger had to be left out of the calculations since he said he was unable to place them; he knew one was "somewhere" on the little finger, but the other seemed "somewhere" on the inner part of the hand.

In seven other patients the investigation of localization was made after protopathic sensibility had made a complete recovery, but before there was any reduction in the loss of epicritic sensibility. The results of this set are recorded in Table IV.

Table IV.-Localization (Pressure) Test, after Complete Nerve Division asd Suture, with Recovery of Protopathic Semsibility at Time of Observation.

\begin{tabular}{lllcc} 
Patient & \multicolumn{1}{c}{ Nerve } & \multicolumn{1}{c}{ Site of suture } & $\begin{array}{c}\text { Average error for } \\
\text { localization over area } \\
\text { of epicritic loss }\end{array}$ & $\begin{array}{c}\text { Average error for } \\
\text { localization over } \\
\text { normal area }\end{array}$ \\
B. K. & Median and ulnar & Wrist & $\mathbf{1} \cdot 4$ & $\cdot 9$ \\
A. N. K. & Ulnar & Elbow & $1 \cdot 4$ & $\cdot 5$ \\
S. O. & Ulnar & Middle of forearm & $1 \cdot 4$ & $\cdot 5$ \\
J. R. M. & Ulnar & Upper forearm & $1 \cdot 6$ & $\cdot 5$ \\
O. R. & Median & Middle of forearm & $1 \cdot 4$ & $\cdot 8$ \\
V. P. & Ulnar & Lower forearm & $3 \cdot 0$ & $\cdot 5$ \\
T. T. P. & Median & Lower forearm & 1.1 & $\cdot 5$
\end{tabular}

The average error for the seven patients over the area of epicritic loss was $1.6 \mathrm{~cm}$. and over the normal part $0.6 \mathrm{~cm}$. 'This series shows that the error in localization is not diminished during the recovery of protopathic sensibility. In two additional patients considerable time had elapsed since the performance of the suture and some recovery of epicritic sensibility had occurred, as well as the return of protopathic sensibility. In both patients gross errors were found with the compass test over the cutaneous distribution of the nerve, although this was sensitive to the lightest application of a camel hair-brush. The findings of these two patients are shown in Table V.

It is of great interest to notice that in both patients the remaining 
deficiency in sensation affected discrimination, localization, and the recognition of posture and passive movement in the joints supplied by the nerve.

Table V.-Localization (Pressure) Test, after Complete Nerve Dirision and Suture, with Recovery of Protopathic and partial Recovery of Epicritic Sensibility at Time of Observation.

$\begin{array}{lllcc}\text { Patient } & \text { Nerve } & \text { Site of suture } & \begin{array}{c}\text { A verage error for } \\ \text { localization over area } \\ \text { of partial epicritic loss }\end{array} & \begin{array}{c}\text { A verage error for } \\ \text { localization over } \\ \text { normal area }\end{array} \\ \text { W. H. } & \text { Ulnar } & \text { Arm } & 1 \cdot 0 & \cdot 7 \\ \text { T. N. W. } & \text { Median } & \text { Upper forearm } & 1 \cdot 3 & 9\end{array}$

In one other patient the only evidence of any regeneration of afferent fibres was a slight recession of protopathic loss, although four years had elapsed since the time of the secondary suture of the median, and the error in localization for the anæsthetic area was $1.2 \mathrm{~cm}$., whereas for the normal part it was $0.4 \mathrm{~cm}$.

By the application of this method of testing localization, which gives measurable results, to patients known to be suffering from division of peripheral nerves, it is shown that, contrary to the present view, accurate tactile localization is not present even when the pressure is sufficient to stimulate the deep afferent system. It is difficult to decide whether the defective power of localization in these twenty patients was due to loss of cutaneous or subcutaneous nerve terminals, or both, since pressure may stimulate both, and although in eighteen of the patients the skin was anæsthetic, we have no accurate anatomical or physiological information about the fibres of the median or ulnar distributed to subcutaneous tissues.

Experiment 6.-Head [5] has stated that "localization is in all probability the sum of two sets of sensation, one of which arises from deep, the other from cutaneous stimulation." The foregoing experiments are in perfect agreement with this contention, but it is difficult to decide whether the errors in localization in Experiment 5 were due to the loss of cutaneous sensation only, or to a disturbance of the deep system in addition. The power of localization which remains, in the patients used in Experiment 5, must be entirely dependent upon the deep system, but for the anatomical reasons stated in an earlier section, we cannot claim that it represents the normal power of localization served by fibres terminating in subcutaneous tissues. In an endeavour to arrive more closely at a correct conception of the part played by the cutaneous and deep systems, several preparations were applied to the unbroken skin in an attempt to obtain an uncomplicated cutaneous 
anæsthesia. None of these gave a satisfactory anæsthesia, until on the suggestion of my colleague, Professor R. B. Wild, a 10 per cent. solution of cocaine hydrochloride in chloroform was used. With this it was generally found practicable to get an almost complete cutaneous anæsthesia of a small area in about ten to fifteen minutes. The solution was applied to the dorsum of the left index finger in three of the subjects used in Experiment 3. When the skin was found, by testing with a wisp of cotton wool, to be satisfactorily anæsthetized the examination of localization was made by eight pressure stimulations. At a later date the normal power of localization of the same part was tested by eight pressure stimulations. The average error for the eight stimulations, in the three subjects with and without cutaneous anæsthesia, are shown in Table VI.

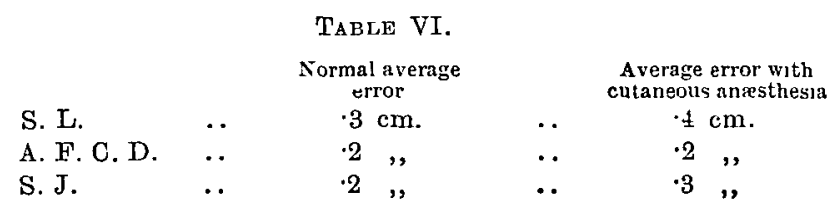

It will be seen that in the three subjects the power of localization was almost as accurate when there was cutaneous anæsthesia as normally. It is only possible to anæsthetize satisfactorily a small cutaneous area and this may be raised as a criticism, but although only the dorsum of one finger was examined the result of the experiment strongly favours the presumptions that:-

(1) Accurate localization is possible through the deep system alone.

(2) Since the errors in localization in the twenty patients examined in Experiment 5 were gross, there was a disturbance of localization through the deep system and the errors were not dependent upon a loss of cutaneous localization.

In several of the patients examined in Experiment 5 the division of the nerve occurred at the wrist or in the lower part of the forearm, and there was an average error of twice the normal. Therefore we may suggest with some confidence that accurate localization through the deep system alone is disturbed by a section of the median or ulnar at the wrist.

\section{V.-Pressure Pain.}

To investigate deep sensibility further it was necessary to test that form of sensation which is now well known as pressure pain.

For this purpose an algometer was made for me similar to that used 
by Rivers [3]. From some preliminary tests with the instrument it became clear that certain technical difficulties in its use were to be overcome, and it appeared necessary to carry out at the outset some experiments upon normal individuals in order to establish a standard procedure when using the instrument upon patients.

Experiment 1.-These preliminary tests were made upon six normal individuals, three on the right hand and three on the left, and in each the pressure was applied at eight selected points which are shown in fig. 2. At each point three readings were taken, and it was soon found

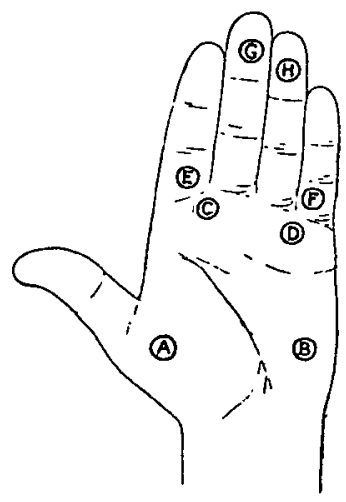

FIa. 2. - To show the position of the eight selected points used in Experiment 1 (pressure pain).

that the second and third readings ought not to be taken over the exact point of the former, since repetition of pressure over the same spot resulted in pain being experienced with much less pressure than on the previous occasion. Inequality in the three records in the same neighbourhood was found to be due to differences in the rate of application of the pressure; it was therefore necessary to apply the pressure evenly and slowly, which is by no means easy, and some of the greater differences in the three readings may be due to this cause. A third cause of difficulty was due to the personal factor, as it was not always possible to get the person examined to indicate at once the onset of the painful element in the sensation aroused by pressure. In the first series, several informed me that there was a great tendency to compare a second experience of pain with the first, which tended to disturb their judgment. These sources of fallacy make it important, as Head as already stated, to take at least three readings at each point. 

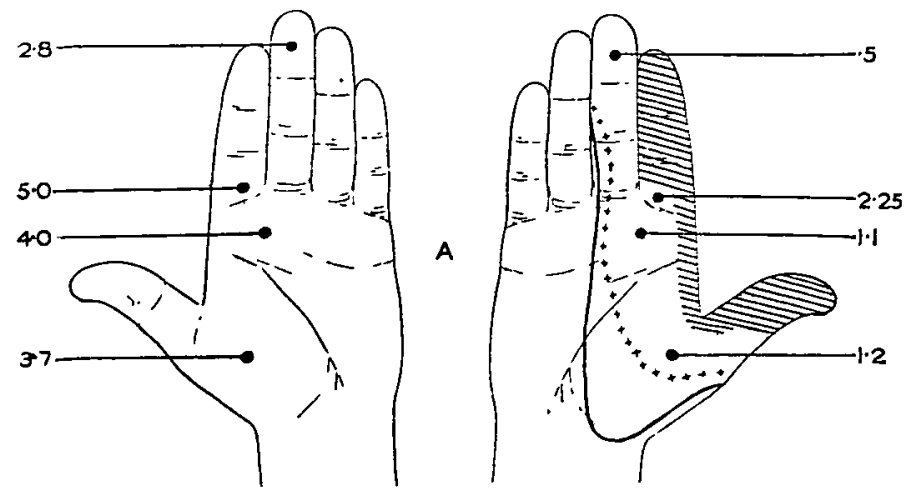

Frg. 3, A.-Division of median nerve in arm.
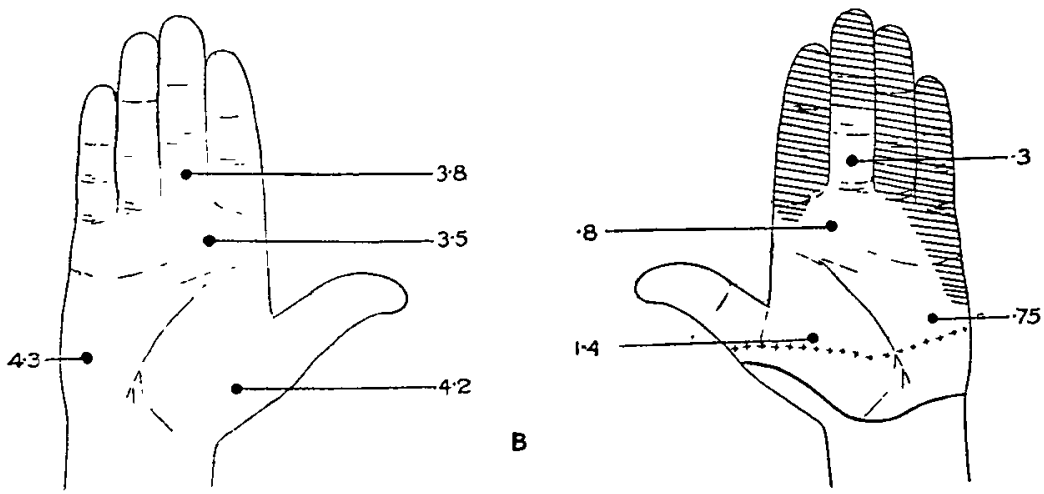

FIG. 3, B.-Division of median and ulnar nerves in arm.

Crosses = analgesia to prick of pin.

Thick line $=$ anæsthesia to lightest applications of fine brush.

Shading = complete sensory loss.
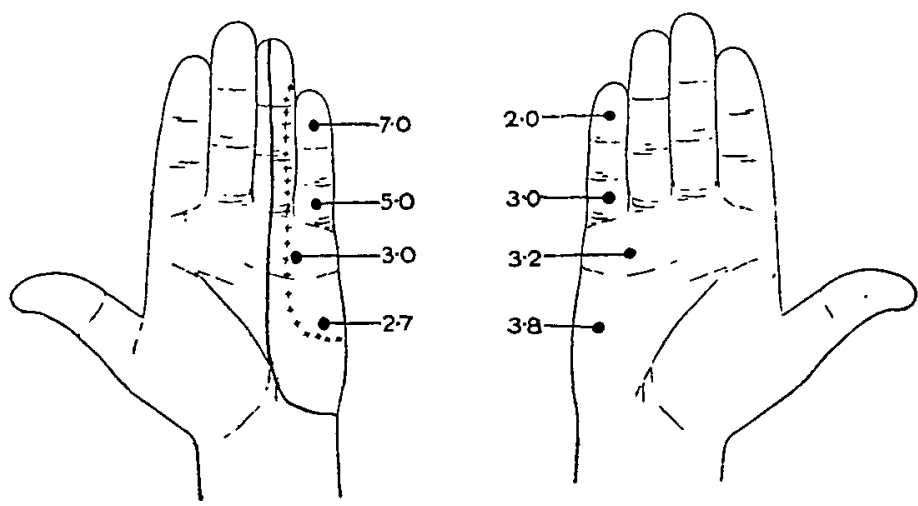

FIG. 4.-Division of ulnar nerve in upper forearm.

Crosses $=$ analgesia to prick of pin.

Thick line $=$ anæsthesia to lightest applications of fine brush. 
The results of the investigation of the six normal individuals are given in the Table VII, which shows all the three readings at each of the eight points.

Tabe VII.-To show the Amodnt of Pressure required to induce Pain, at Eight Selected Poists on the Hand and Digits, in Six Normal Individuals.

\begin{tabular}{|c|c|c|c|c|c|c|c|c|}
\hline S. J. & $\begin{array}{c}\text { A } \\
12.50 \\
2.75 \\
12.50\end{array}$ & $\begin{array}{c}\text { B } \\
3 \cdot 25 \\
3 \cdot 00 \\
3 \cdot 50\end{array}$ & $\begin{array}{c}\mathrm{c} \\
1.50 \\
1.25 \\
1.25\end{array}$ & $\begin{array}{c}\text { D } \\
1 \cdot 25 \\
1 \cdot 25 \\
1 \cdot 50\end{array}$ & $\begin{array}{c}\mathbf{E} \\
3 \cdot 00 \\
2 \cdot 00 \\
2 \cdot 50\end{array}$ & $\begin{array}{c}\mathbf{F} \\
2 \cdot 50 \\
2 \cdot 50 \\
2 \cdot 50\end{array}$ & $\begin{array}{r}G \\
1.00 \\
\cdot 75 \\
1.25\end{array}$ & $\begin{array}{c}\mathbf{H} \\
.75 \\
.75 \\
1.00\end{array}$ \\
\hline M. D. & $\left\{\begin{array}{l}2 \cdot 50 \\
2 \cdot 00 \\
1 \cdot 75\end{array}\right.$ & $\begin{array}{l}2 \cdot 50 \\
2 \cdot 25 \\
1 \cdot 75\end{array}$ & $\begin{array}{l}1.50 \\
1.25 \\
2.00\end{array}$ & $\begin{array}{l}.50 \\
.50 \\
.75\end{array}$ & $\begin{array}{l}2 \cdot 25 \\
1 \cdot 00 \\
1 \cdot 25\end{array}$ & $\begin{array}{l}2 \cdot 00 \\
1 \cdot 50 \\
1 \cdot 75\end{array}$ & $\begin{array}{l}.50 \\
.75 \\
.25\end{array}$ & \\
\hline D. S. & $\left\{\begin{array}{l}2 \cdot 75 \\
2 \cdot 00 \\
100\end{array}\right.$ & $\begin{array}{l}2 \cdot 00 \\
2 \cdot 50 \\
2 \cdot 50\end{array}$ & $\begin{array}{r}1.25 \\
1.00 \\
\cdot 75\end{array}$ & $\begin{array}{r}1 \cdot 00 \\
\cdot 75 \\
\cdot 75\end{array}$ & $\begin{array}{l}1 \cdot 00 \\
1 \cdot 25 \\
1 \cdot 50\end{array}$ & $\begin{array}{r}1 \cdot 00 \\
.75 \\
\cdot 75\end{array}$ & $\begin{array}{l}\cdot 25 \\
-25 \\
.25\end{array}$ & \\
\hline R. F. & $\left\{\begin{array}{l}2 \cdot 25 \\
2 \cdot 25 \\
1 \cdot 75\end{array}\right.$ & $\begin{array}{l}2 \cdot 25 \\
1.00 \\
1.75\end{array}$ & $\begin{array}{l}1 \cdot 25 \\
1.50 \\
1 \cdot 00\end{array}$ & $\begin{array}{l}1 \cdot 00 \\
1 \cdot 25 \\
1 \cdot 00\end{array}$ & $\begin{array}{l}1.75 \\
1.75 \\
1.50\end{array}$ & $\begin{array}{l}2 \cdot 00 \\
1 \cdot 50 \\
1 \cdot 25\end{array}$ & $\begin{array}{l}2 \cdot 00 \\
2 \cdot 25 \\
1 \cdot 75\end{array}$ & \\
\hline L. S. & $\left\{\begin{array}{l}1 \cdot 50 \\
1 \cdot 75 \\
175\end{array}\right.$ & $\begin{array}{l}2.00 \\
2.00 \\
1.50\end{array}$ & $\begin{array}{l}.75 \\
.75 \\
.75\end{array}$ & $\begin{array}{r}1.00 \\
.50 \\
1.00\end{array}$ & $\begin{array}{r}1 \cdot 25 \\
1 \cdot 50 \\
\cdot 75\end{array}$ & $\begin{array}{r}.75 \\
1.50 \\
1.25\end{array}$ & $\begin{array}{l}.50 \\
.50 \\
.25\end{array}$ & \\
\hline H. G. & $\left\{\begin{array}{l}2 \cdot 75 \\
2 \cdot 50 \\
2 \cdot 75\end{array}\right.$ & $\begin{array}{l}3 \cdot 50 \\
3 \cdot 00 \\
3 \cdot 25\end{array}$ & $\begin{array}{l}1 \cdot 50 \\
1.50 \\
2 \cdot 25\end{array}$ & $\begin{array}{l}2 \cdot 25 \\
2 \cdot 25 \\
2 \cdot 75\end{array}$ & $\begin{array}{l}2 \cdot 75 \\
2 \cdot 50 \\
2 \cdot 75\end{array}$ & $\begin{array}{l}2 \cdot 50 \\
2 \cdot 75 \\
2 \cdot 75\end{array}$ & $\begin{array}{l}2 \cdot 25 \\
2 \cdot 00 \\
2 \cdot 00\end{array}$ & $\begin{array}{l}2 \cdot 6 \\
2 \cdot 5 \\
2 \cdot 5\end{array}$ \\
\hline
\end{tabular}

The results of this experiment brought out the following information :-

(1) Greater pressure was usually required to produce pain in the thenar and hypothenar regions than at the other points tested.

(2) Less pressure was required at the base of the clefts between the fingers than over the palmar surface of the proximal segment of the digits. This is presumably due to the exposed position of the digital nerves as they divide in the region of the clefts into branches for the adjacent sides of the neighbouring fingers.

(3) With a single exception, less pressure was needed to induce pain over the palmar surface of the distal segment of a digit than over its proximal segment.

Experiment 2.-With the knowledge and experience gained from this preliminary investigation of pressure pain, the test was applied to a number of patients suffering from division of the median or ulnar nerves. To simplify the presentation of the results of this experiment only the average of the three readings at each point is recorded, and for ease of comparison the results of five typical tests are represented in a diagrammatic form (figs. 3, 4 and 5). As in the former experiment the pressure required to induce pain is recorded in kilogrammes. 

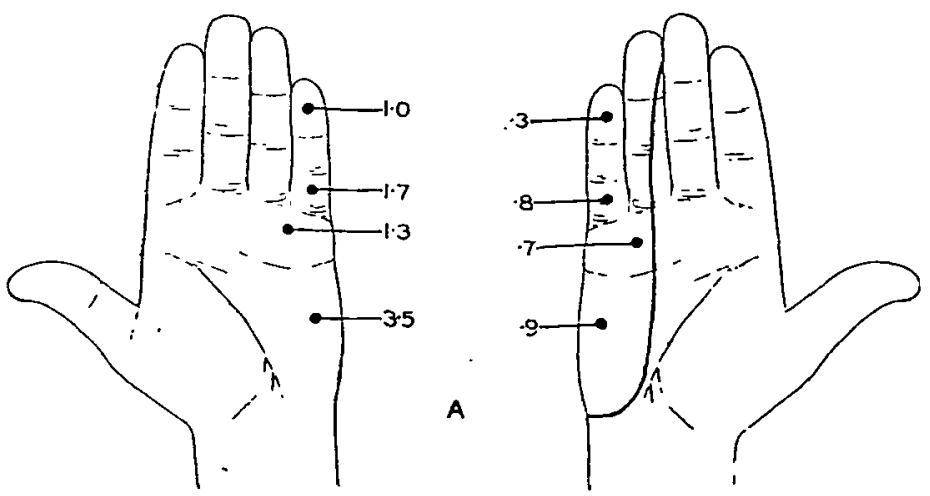

FIG. 5, A.-Division of ulnar nerve in lower forearm.

Thick line indicates anæsthesia to lightest applications of fine brush.
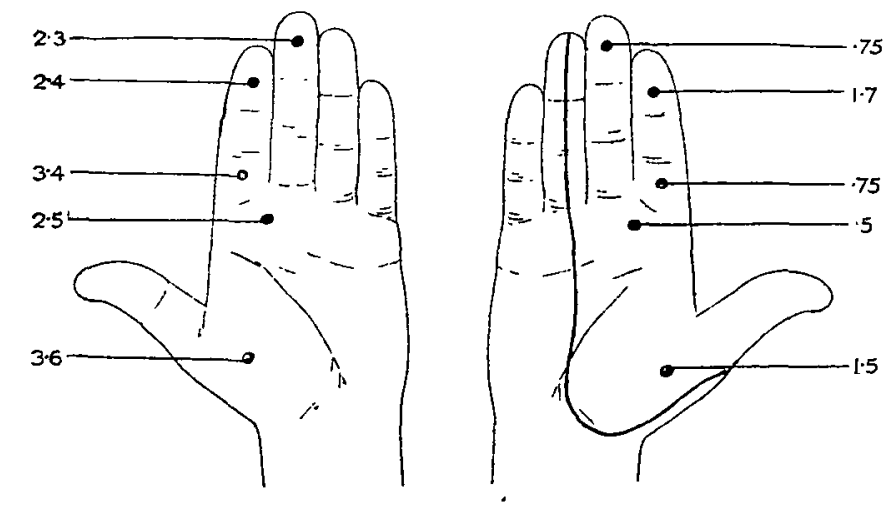

FIG. 5, B.-Division of median nerve in arm.

Thick line indicates anæsthesia to lightest applications of fine brush.

By these experiments it was found :-

(1) That it was distinctly easier to test pressure pain on the abnormal than on the sound side. This was due to the fact that when testing over an area where epicritic sensibility was lost, the patients could more readily recognize the onset of the painful element since it commenced abruptly and rapidly became intensely painful. Several patients told me that they had no doubt on the affected side, whereas on the sound side they were often in difficulty as to when the pressure became actually painful. As Head found, several expressed fear at the application of pressure on the affected side, and this, together with the 
experience that on this side the pain quickly became intense, may have caused in some of the tests an abnormally low reading on the affected side.

(2) In those patients where the division of the nerve was in the distal part of the limb and there was no absolute sensory loss, the readings on the affected side tended to be definitely lower than on the sound side.

(3) In some patients with division of the median in the middle of the forearm, i.e., proximal to the supply of that portion of the flexor sublimis digitorum which flexes the index finger and distal to the origin of the anterior interosseous nerve and branches for the supply of the main flexor mass, considerably more pressure was aequired on the index on the affected than on the sound side. These results pointed to a disturbance, but not complete loss, of this form of sensation in the index after division of the median proximal to the origin of the most distal branch to the flexor sublimis digitorum, and indicated that some fibres for this form of sensation in the index were transmitted by this muscular branch, and probably extended to the digit along the tendon. Similarly, after division of the ulnar in the upper part of the forearm, although the inner part of the flexor profundus digitorum was capable of voluntary action, higher readings were sometimes found on the little finger on the affected side than on the sound side. It is not so easy to give an anatomical reason for this occurrence, which is found only in a proportion of cases of division of the ulnar in the forearm and is nothing like so constant as the similar condition just described in the case of the median, but it may be due to the occasional appearance of an extra branch to the flexor profundus digitorum in the lower part of the forearm.

The associated demonstrable disturbance of the appreciation of contact in these cases will be referred to in the next section.

(4) In the patients examined during recovery, when there was a complete return of protopathic sensibility (fig. 5), the readings at all points on the affected side were lower than on the normal side. This was found even over digits that at the outset had exhibited an absolute sensory loss, or which originally had given a higher reading. These results indicate that the recognition of pressure pain returns at an early stage in recovery, at the same time as protopathic sensibility. It is also significant that in cases of only partial recovery of sensation pressure pain like protopathic sensibility is one of the most constant to manifest some improvement. 


\section{VI.-Recognition of the "Contact" of Tactile Pressure.}

In routine examinations of patients suffering from injury to, or disease of, the peripheral nerves, this aspect of deep sensibility is usually the only one tested. Consequently if the patient appreciates everywhere the contact of some instrument, as the head of a pin, "no loss of deep sensibility" is generally recorded in the notes. It does not seem to have been realized previously that the various forms of sensation grouped together as deep sensibility may be dissociated, and this, together with the widespread belief that deep sensibility is practically entirely transmitted by afferent fibres which run with motor nerves and reach their termination by accompanying such structures as long tendons, has been responsible for the confidence with which observers have been content to investigate simply the recognition of contact as the standard test for deep sensibility.

As is to be expected this form of deep sensibility is only appreciably affected in lesions in the proximal part of the limb, i.e., above the origin of the supply of branches to the long flexors of the digits. There is no - doubt that mere recognition of contact is chiefly transmitted by afferent fibres which run in the branches to the long flexor muscles of the digits. In this respect it is instructive to refer to the fairly constant occurrence of complete anæsthesia of the distal part, and sometimes the whole of the palmar surface of the index finger after complete division of the median in the middle third of the forearm, although the division occurs distal to the origin of the main branches supplying the flexor sublimis digitorum and the anterior interosseous nerve. This is apparently due to the origin from the median trunk in the lower third of the forearm of a branch which supplies that portion of the flexor sublimis digitorum which flexes the proximal interphalangeal joint of the index finger. From a study of the number of patients with division of the median in the upper part of the forearm, where the precise anatomical level with regard to the origin of branches had been defined at operation, I am inclined to the view that a greater proportion of afferent fibres pass in the branches to the flexor sublimis digitorum than to the flexor profundus digitorum.

From personal observations it seems to be almost a rule that if any power to appreciate contact is retained in a part there is always in the same region some retention of the power to recognize pressure pain, although the amount of pressure required to induce pain may be very much greater than in the same region on the sound side. This latter 
point is well illustrated by the following record, which also supports the previous contention that more afferent fibres are transmitted by the tendons of the flexor sublimis digitorum than those of the flexor profundus digitorum.

Patient, J. C. Complete division of the ulnar was found in the upper part of the forearm, just distal to the origin of branches for the supply of the flexor carpi ulnaris and inner half of the flexor profundus digitorum, which muscles possessed voluntary power. The loss of epicritic and protopathic sensibility corresponded to the full typical normal distribution. It was impossible to measure the error in the localization of tactile pressure over the little finger, as the best response to any stimulation he gave was "somewhere on my little finger," and he was quite unable to attempt to chart any of the stimulations in this region, although he invariably recognized that a contact had been made, provided sufficient pressure was used. The average of the three readings with the algometer on the two sides at four points may be conveniently tabulated for comparison.

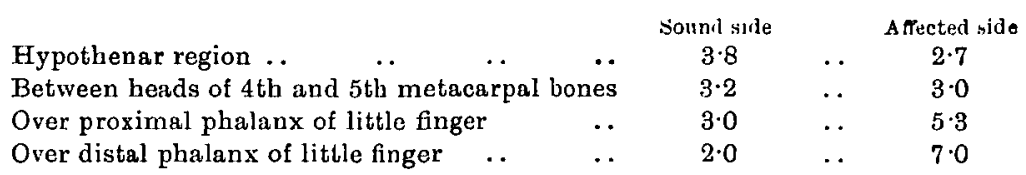

It will be noticed that in the first two regions the reading was slightly lower on the affected side, whilst in the case of the two tests on the little finger the reading was markedly higher on the affected side, being more than three times greater on the distal phalanx.

Two other patients in whom similar results were found have been encountered. I have never seen "contact" and "pressure pain" completely dissociated. On the other hand these two forms of deep sensibility may be retained in a part without any power of localization being present, as in the little finger of the patient just referred to.

After division of the ulnar nerve at any point proximal to the origin of the branches for the supply of the flexor profundus digitorum, the area in which complete anæsthesia to all stimuli is found is very constant in extent and affects the little finger and the inner border of the hypothenar region as shown in fig. 6. After division of the median above the level of the branches supplying the long flexor muscles, the area of complete anæsthesia is by no means so constant. In sixteen cases of known complete division of the median, at or proximal to the level of the elbow joint, there was complete anæsthesia only of the index and a small neighbouring portion of the palm (fig. 7, A) in six; of the index and medius and a larger portion of the palm in five 
(fig. 7, в) ; and the index, medius and palmar surface of thumb together with a still larger extent of the palm in five (fig. $7, \mathrm{c}$ ). The infrequency (only five out of sixteen) of complete anæsthesia of the palmar surface of the thumb was rather surprising, but the foregoing results were confirmed by the examination of a group of patients suffering from

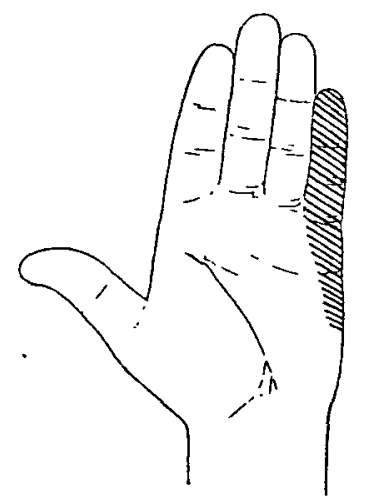

FIG. 6.-To show the typical extent of absolute sensory loss after division the ulnar nerve in the arm.
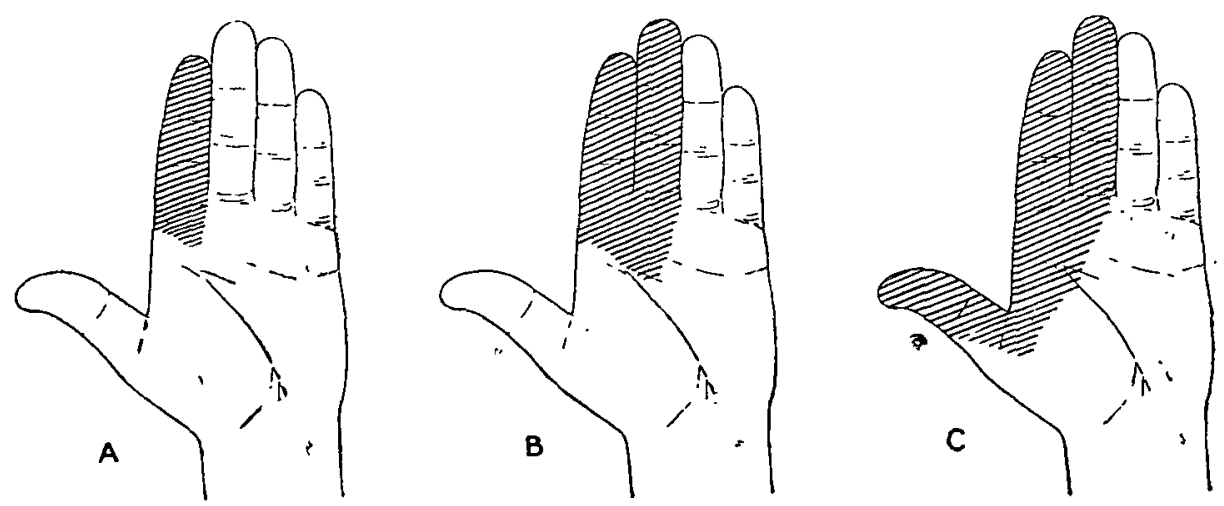

FIG. 7.-To show the variation in extent of the absolute sensory loss after division of the median nerve in the arm.

division of both the median and the ulnar nerves in the upper arm, in whom contact (if sufficient pressure was made) was recognized over the palmar surface of thumb in an even higher proportion. Consequently it must be surmised that the radial nerve, and possibly in a small number the musculo-cutaneous, by means of perforating branches, takes 
part in the innervation of the deeper tissues of the palm and the palmar surface of the thumb.

\section{VII.--Summary and Conclusions.}

In each section the main conclusions which may be drawn from the experiments and investigations are presented; it now only remains necessary to restate the principal conclusions bearing upon our present conception of sensation, and these may be conveniently summarized in three divisions.

(1) It seems clear that no nerve of sufficient size to be of clinical importance, or to be selected for experimental work upon sensation, is distributed exclusively to skin. Consequently after section of any of the nerve trunks, even in the distal part of a limb, there is bound to be some disturbance of so-called deep sensibility, and it seems quite impracticable to divide sensation into superficial and deep. Besides this anatomical criticism of such a division there is to be remembered the additional confusion which may arise from the knowledge that one form of sensation at least is transmitted by both cutaneous and deep nerves. It has been proved that so-called deep sensibility is not transmitted entirely by nerve fibres which accompany muscular. branches, since the sensory supply of the joints of the fingers and thumb undoubtedly arises from the digital nerves, and it seems probable that other forms of deep sensibility are in part, at least, transmitted by branches arising independently from the main nerve.

(2) The various forms of sensation included under the division of deep sensibility may be dissociated. After division of the median at the wrist there is found a loss of the recognition of passive movements at the metacarpo-phalangeal and interphalangeal joints of the digits supplied by the nerve: some power of localization is retained over the region of the cutaneous distribution of the nerve, although by means of a measurable test a gross error is seen on comparing the results with those obtained on the normal part; the least pressure is readily recognized everywhere, and the readings with an algometer show that rather less pressure is required to induce pain on the affected than on the sound side. If division of the median occurs proximal to the supply of the outer part of the flexor sublimis digitorum in the lower third of the forearm, in addition to the defects just recorded there is usually a complete sensory loss to all forms of stimulation over the distal two segments, or the whole of the index finger. In a small 
proportion after section of the median at this level the loss of sensation in the index finger is less profound. Although in these there is no power to localize, the contact of pressure can be recognized and pain can be induced by excessive pressure, but on investigating the two latter forms of sensation more fully it is discovered that more pressure is required to induce the sensation of contact over the index than the medius and the reading with the algometer is considerably higher than on the sound index. The condition of what we know as deep sensibility in the index is, in such cases, in a most interesting stage and may be summarized as follows :-

(i) Loss of sense of position and recognition of passive movement in all joints of this finger.

(ii) Absolute loss of localization.

(iii) Retention of recognition of contact and pressure pain, but on the application of measurable tests it is found that these forms of sensation are grossly affected.

Little reference need be made to the effect upon deep sensibility of a lesion at a higher level, in the region of the elbow or in the arm, since, except with regard to localization which has already been fully discussed, the condition is well known; reference is only necessary to the fact that the extent of the absolute sensory loss to all stimuli is variable.

(3) Observations upon the recovery of deep sensibility demonstrate several striking and significant features. Reappearance of the recognition of contact and appreciation of pain on the application of excessive pressure occurs early, as a rule during the stage of protopathic recovery. At first considerable pressure is required before it is appreciated, but the recovery of contact without concomitant recovery of pressure pain has never been encountered, although the extremely high reading with the algometer in two patients examined suggest that this is possible, and there does not appear to be any obvious reason why these two forms should not be dissociated. As regeneration proceeds less pressure is found to be required at subsequent examinations. As the recognition of contact and pressure pain improve there is usually a return of some power of localization, but it is very grossly defective, the error being much greater in regions where there has previously been an absolute sensory loss than elsewhere. Further improvement in localization and signs of recognition of passive movement of joints occurs very late, and not until there is evidence of recovery of epicritic sensibility. Most of my studies have 
been upon patients who have had a secondary suture of the median or ulnar nerve, and under these circumstances any recovery of accurate localization or recognition of passive movements of joints is at the best very defective and frequently remains absent. After primary suture there is a better opportunity for studying the later phases of recovery. It seems very significant that recovery of recognition of contact and appreciation of pressure pain, followed often by a gross and defective return of the power of localization, occur early during the stage of reappearanee of protopathic sensibility, whilst accurate localization and recognition of passive movement only appear late, at a time when there is also evidence of epicritic return as tested by graduated hairs and the compass. That is to say the three forms of sensation concerned with spacial recognition-accurate localization, discrimination and recognition of passive movement-only recover at a very late stage, and even after primary suture are apt to remain defective.

These observations upon the recovery of certain forms of sensation after suture of a peripheral nerve suggest two important facts.

(a) What we have regarded as deep sensibility ought to have been subdivided, just as cutaneous sensation has been divided, into epicritic and protopathic. There is no doubt that the two forms of sensation, recognition of the contact of pressure and appreciation of passive movement, must be subserved by nerve fibres terminating in the deep tissues; the former of these returns early at a time when protopathic sensibility is recovering, whilst the latter returns at a late stage, if at all, during the time of epicritic recovery. The mere recognition of the contact of pressure is a crude form of sensation compared with the more highly graded appreciation of the direction, localization and range of passive movement of a joint.

(b) In the light of Head's recent work upon the sensory cortex [2], these results suggest that at the periphery, both in cutaneous and deep tissues, we have cortical and thalamic aspects of sensation. The dissociation which is encountered after the division of any large nerve trunk makes it exceedingly difficult to unravel the fundamental principles upon which sensation at the periphery is based, but when we get an accurate conception of the anatomical distribution, and in the light of this knowledge analyse the results of careful clinical examinations of a number of patients suffering from division of a peripheral nerve, the curious forms of dissociation become more intelligible. Continued examinations of the same patients during recovery seem to bring us 
still nearer to a knowledge of the true state of affairs. It has just been stated that certain forms of deep sensibility constantly recover during the early stage when the area of protopathic loss is found to be receding, whilst other forms do not show any recovery until a late stage when there is clinical evidence of a return of epicritic sensibility. In the examinations of a very large number of patients I have found this to be an invariable rule.

It appears to have been generally believed that the earlier return of protopathic sensibility is dependent upon a more rapid rate of regeneration in protopathic than epicritic fibres. Such an explanation supposes some structural difference in the two systems, and Ranson [4], on histological and experimental evidence, made the suggestion that impulses giving rise to pain travel in non-medullated fibres; but more recent work by Adrian [1] shows that it is highly improbable that there is any definite structural difference in the fibres concerned with protopathic and epicritic sensation. A simpler explanation is suggested when we take into consideration all the forms of sensation which return during the later stage of recovery. The forms appearing late, which more frequently show imperfect recovery, or in quite a proportion of cases of secondary suture fail to recover, are those which Head has proved to have cortical representation; whilst the forms which exhibit early and more constant recovery are those which may be expected to persist after destruction of the sensory cortex. Therefore it seems more reasonable to expect to find an explanation of the two stages of recovery in the central nervous system, rather than to find it due to a different rate of regeneration of two sets of fibres which anatomically appear to be identical. After suture and regeneration of a peripheral nerve a much longer period may be expected to elapse before the fibres subserving cortical forms of sensation function correctly, than in the case of the fibres conducting the thalamic aspects of sensation; since a very much more complex readjustment and re-education must occur after regeneration of the fibres in the former case than in the latter. Furthermore, on the hypothesis suggested in this paragraph, we seem to have ample reasons for the more constant and perfect recovery of such aspects of sensation, as cutaneous pain, pressure pain and appreciation of extremes of temperature, than for the higher forms, as those concerned with spacial recognition.

(The expenses of this research have been borne in part by a grant from the Royal Society.) 


\section{REFERENCES.}

[1] Adrian, E. D. "The Response of Human Sensory Nerves to Currents of Short Duration," Journ. Physiology, 1919-20, 53, 70.

[2] HEAD, H. "Sensation and the Cerebral Cortex," Brain, 1918, 41, 57.

[3] Idem. " Studies in Neurology," vol. i, Part I, London, 1920.

[4] Rarson, S. W. "Unmyelinated Nerve-fibres as Conductors of Protopathic Sensation," Brain, 1915, 38, 381.

[5] Rivers, W. H. R., and Head, H. " A Human Experiment in Nerve Division," Brain, $1908,31,323$.

[6] Stopeond, J. S. B. "The Variations in Distribution of the Cutaneous Nerves of the Hands and Digits," Journ. Anat., 1918, 53, 14.

[7] Idem. "The Results of Secondary Suture of Peripheral Nerves," Brain, 1920, 43, 1.

[8] Idem. "The Nerve Supply of the Interphalangeal and Metacarpo-phalangeal Joints," Joum. Anat., 1921, 56, 1.

[9] Idem. " "Tactile Localization," Journ. Anat., 1921, 55, 249. 